\title{
Outbreak of Infections Caused by Shigella flexneri 2a with ESBL-Producing and Quinolone-Resistance in a Mental Healthcare Center in China
}

Tiantian Lu

Zhejiang Xiaoshan Hospital

Chuanling Zhang ( $\square$ wuhuigt@163.com )

zhejiang xiaoshan hospital

Yan Chen

Zhejiang Xiaoshan Hospital

Yuanyu Guo

Zhejiang Xiaoshan Hospital

Research article

Keywords: Shigella, outbreak, antibiotic resistance, PFGE

Posted Date: August 21st, 2019

DOI: https://doi.org/10.21203/rs.2.13262/v1

License: (c) (i) This work is licensed under a Creative Commons Attribution 4.0 International License.

Read Full License 


\section{Abstract}

An outbreak of bacillary dysentery occurred in the Xiaoshan Mental Healthcare Center from November 8 to 16, 2012. Eight strains of Shigella flexneri 2a (S. flexneri 2a) were isolated from the same ward. The aim of the investigation was to clarify the origin of outbreak. Rectal swabs or fecal specimens from 7 doctors, 15 nurses, 2 nursing workers, 3 canteen workers, as well as 18 swabs from environmental sample in the ward were screened for Shigella. Phenotypic analysis included standard microbiological identification techniques, serotyping and antimicrobial susceptibility testing. PCR and sequencing were used to characterize drug resistance and virulence genes. Pulsed field gel electrophoresis (PFGE) was carried out for all Shigella isolates. S.flexneri 2a with multidrug resistance was isolated from eight patients and one canteen worker, which carried multiple antibiotics resistance genes and virulence determinants. The results of PFGE confirmed that nine strains of S. flexneri 2a belonged to the same clone. It was suggested that the outbreak strain spread directly from person-person or indirectly from

person-food-person from the canteen worker. The outbreak was controlled after quarantine of ill residents, replacement of antibiotics and improvement of hygienic condition.

\section{Background}

Shigella species, which are important pathogens of the gastrointestinal tract, are responsible for causing severe diarrhea and acute gastroenteritis, especially in developing countries. It has been estimated that Shigella causes 165,000 deaths worldwide every year, of which 55,000 are children younger than 5 years of age [1,2]. In mainland China, the incidence of Shigella infections is 20.28 cases per 100,000 people per year [3]. Shigella is transmitted by the fecal-oral route and can cause large outbreaks, thus posing a great threat to human public health [4-9].

Currently, third-generation cephalosporins and fluoroquinolones are the main antimicrobial agents in the treatment of Shigella infection in China; nevertheless, increasing multidrug resistant Shigella has become a serious public health threat [10]. Multidrug resistance to fluoroquinolones, third-generation cephalosporins and azithromycin has been reported worldwide as a growing problem of multidrug resistance in Shigella [11-14].

Here, we report an outbreak of infection caused by S. flexneri 2a with ESBL-producing and quinoloneresistance in Xiaoshan District, Hangzhou, China. From November 8, 2012 to November 16, 2012, eight strains of $S$. flexneri 2a were isolated form 12 psychiatric patients form Xiaoshan Mental Healthcare Center of Zhejiang Province suffering from diarrhea to varying degrees. Patients were isolated, treated and comprehensive measures were taken to control further outbreak. An investigation started on 15 November 2012. Fortunately, the outbreak was effectively controlled in a short period of time and all patients recovered and there were no deaths.

\section{Methods}




\section{Outbreak investigation}

During the outbreak, we firstly isolated all infectious patients who received treatment with antibiotics. Next, we started examining the possible reasons of the outbreak. Since all patients were mentally ill and unable to communicate effectively, all relevant medical data (frequency of diarrhea, dietary process, the patient's clinical manifestations and external contact) were provided by the patients' physicians and nurses. Next, stool sample from patients and personal (doctors, nurses, nursing workers, canteen staff), as well as environmental sample in male wards were screened for Shigella. The ethics committee of the Mental Healthcare Center approved this study.

\section{Laboratory investigation}

Stool specimens from all patients with diarrhea were cultured for the detection of enteric pathogens. Suspected isolates were identified by VITEK2 system (BioMérieux, France) and the serotype of Shigella isolates were confirmed by slide agglutination using commercial antisera (Denka Seiken Co. Ltd, Tokyo, Japan), following the manufacturer's recommendations. Antimicrobial susceptibility testing of the Shigella isolates was performed by broth microdilution method and the susceptibility was interpreted according to Clinical and Laboratory Standards Institute guidelines [21]. Polymerase Chain Reaction(PCR) and sequencing were used to characterize topoisomerases ( $g y r A$, gyrB, parC, parE) in the quinolone-resistance determining regions (QRDRs) and the ESBL genes (bla TEM bla ${ }_{\text {OXA }}$, bla CTX $_{\text {M }}-1,2,8,9$ and $\left.b / a_{S H V}\right)$ and virulence determinants (ial, iapH, set1A, set1B, sen and virA) [14,22]. Pulsed-field gel electrophoresis (PFGE) after the Xbal digestion of chromosomal DNA was carried out for all Shigella isolates [23].

\section{Results}

\section{Epidemiologic investigation}

Eight strains of $S$. flexneri 2a were isolated from fresh fecal specimens of 12 patients (average age $48.9 \pm 18.9$ years old; youngest patient was seventeen and the oldest one was seventy-four years old) with diarrhea from the same ward of Xiaoshan Mental Healthcare Center in Hangzhou, China. The Mental Healthcare Centre is a fully enclosed medical and rehabilitation institution, which is divided into male ward, female ward and intensive care area. The outbreak occurred in the male ward with twenty-five patients; twelve patients had different degrees of diarrhea accompanied by high temperature $\left(>38^{\circ} \mathrm{C}\right)$ with blood-free mucus feces. Finally, a total of eight Shigella strains were isolated from twelve samples and the serotype of all isolates were flexneri $2 a$.

The investigation showed that no doctors, nurses or nursing workers were infected with Shigella. Interestingly, S. flexneri 2a was detected in stool sample of a canteen staff worker (his job was to distribute food to patients in the male wards). The staff member suffered from diarrhea and abdominal pain. Those symptoms accrued before the outbreak in patients, which suggested the worker was likely to 
be a source of infection. Moreover, the serotype, drug sensitivity, resistance and virulence genes of the strain were the same as that in the Shigella presented in the outbreak.

Furthermore, in March 2013, we isolated the S. flexneri 2a from feces of two patients who have been infected with Shigella. In February 2014, one S. flexneri 2a was isolated from patient with initial infection. Then we confirmed that these isolates belong to the same clone as the outbreak strains. After that, we continued the monitoring for three years, except for four patients who had been discharged from the hospital. The investigation ended in December 2016. This phenomenon suggests that patients infected with Shigella do not have long-term immunity; it may turn into a latent infection under the intervention of antibiotics, and relapse when the body's immune system decreases.

\section{Laboratory results}

All 8 S. flexneri 2a isolates were resistant to ampicillin, trimethoprim/sulfamethoxazole, tetracycline, cefotaxime, ceftazidime, cefepime, ciprofloxacin, but were sensitive to piperacillin/tazobactam, imipenem, and the MIC of azithromycin was $2 \mu \mathrm{g} / \mathrm{mL}$ (no interpretation standard) (Table 1). Moreover, all strains produced extended-spectrum $\beta$-lactamase (ESBL). In addition, the PCR and sequencing results showed that all outbreak isolates carried bla ${ }_{C T X-M-57}, b / a_{O X A-30}, b / a_{T E M-1}$ resistant genes and ia 1, ipaH, set/B, sen and virA virulence genes. Meanwhile, the gyrA (Ser83Leu) and parC (Ser80lle) amino acid mutations were discovered in quinolone resistance determining regions (QRDRs) (Table 2). Also, the PFGE results showed that all 9 S. flexneri $2 a$ isolates (including the strain isolated from canteen staff) had the same band patterns, which indicated that they belong to the same clone (Figure 1).

\section{Control measures}

During the outbreak of the epidemic, the hospital took emergency preventive and control measures, including patient isolation, environmental disinfection, propaganda and education on hand hygiene, sanitary management of food and water, improvement of ventilation conditions, medical observation of close contacts.

All patients infected with $S$. flexneri $2 a$ were treated with antibiotics. At the initial stage of infection, 3 patients were treated with intravenous cefotaxime (3.0gV/BID), 3 patients with oral norfloxacin $(0.2 \mathrm{~g} / \mathrm{TID}), 1$ patient with oral sulfamethoxazole ( $2 \mathrm{~g} / \mathrm{BID})$ and 1 with intravenous piperacillin/tazobactam $(3.375 \mathrm{~g} / \mathrm{Q} 8 \mathrm{H})$. However, five patients failed to receive effective treatment. Because the S. flexneri $2 a$ isolates produce ESBL and quinolone-resistance, we recommended piperacillin/tazobactam or azithromycin as the main treatment approach for those patients. Consequently, the antibiotics were substituted with intravenous piperacillin/tazobactam $3.375 \mathrm{~g} / \mathrm{Q} 8 \mathrm{H}$ and intravenous cefoperazone/sulbactam 3.0g/BID. All the infected patients were cured and five consecutive cultures of Shigella revealed to be negative.

\section{Discussion}


This study investigated a small-scale outbreak of S. flexneri $2 a$ infection in a Xiaoshan Mental Healthcare Center in Hangzhou, China. From March 2012 (observed outbreak) to December 2016 (end of the investigation), 12 strains of $S$. flexneri $2 a$ were isolated, including the infection of a canteen worker and the secondary infection of three patients. After a series of investigations, the canteen staff was identified as the source of Shigella infection (direct contact with patients or food intended for patients). All strains were the same clone type, showing ESBL-Producing and Quinolone-Resistance as well as the same PFGE bands. Because of the multi-drug resistance of the strain, most patients failed to respond to an initial antibiotic treatment and underwent a longer course of treatment. Fortunately, no patients died following episodes of shigellosis.

Laboratory results showed that these outbreak isolates carry multiple antibiotics resistance genes, including CTX-M gene. So far, at least 109 variants of CTX-M enzymes (CTX-M-1 to 124) have been described. Among these enzymes, some exhibit increased hydrolysis activity against ceftazidime, while others display a significantly higher rate of hydrolysis of cefotaxime than ceftazidime [15]. The presence of D240C mutations in $b / a_{C T X-M-57}$ in this study resulted in the increased hydrolysis activity against ceftazidime [16]. Besides, the outbreak $S$. flexneri $2 a$ isolates carried a $b / a_{O X A-30}, b / a_{T E M-1}$ genes and conferred resistance to cefotaxime, ceftazidime and cefepime. The main mechanism of quinolone resistance in the Shigella spp. was the mutation of gyrA, such as at codon 83 or 87 and of parC at codon $80[17,22]$. So, the resistance of ciprofloxacin and levofloxacin may be caused by the mutation of gyrA (Ser83Leu) and parC (Ser80lle) in the outbreak isolates.

The ability of Shigella spp. to cause shigellosis is attributed to the expression of arrays of virulence genes associated with colonization, invasion/penetration and toxin-mediated disease, such as the invasionassociated locus (ial), the invasion plasmid antigen $\mathrm{H}$ gene (ipaH), Shigella enterotoxin 1 (ShET-1) gene ( $\operatorname{set} 1 A$ and set1B), Shigella enterotoxin 2 (ShET-2) gene (sen) and virA gene [18-20]. Estimating the existence of virulence determinants in Shigella would help us to further understand its pathogenicity. The $S$. flexneri $2 a$ isolates harbored virA, ial, ipaH, set1B, sen virulence genes, which indicated that the strain possesses a strong virulence, which in turn can cause diarrhea and abdominal pain.

This study has several limitations. First, although it has been determined that the outbreak was transmitted by a canteen staff personnel who distributed food to patients in the male ward, the source of infection for the staff remains unclear due to limited information. Second, due to the lack of in-depth investigation, it is not clear whether workers introduced Shigella into the community before isolation thus causing infection among residents.

\section{Conclusions}

This is the first report on the outbreak of bacillary dysentery in a psychiatric ward in China. Our data suggested that the outbreak was caused by an ESBL-Producing and Quinolones-Resistance S. flexneri $2 a$ strain which was introduced into the Mental Health Center by a canteen staff who distributed food to patients in the male wards. It was likely that the outbreak strain spread via direct person-person or indirect 
person-food-person mode of transmission. Our findings suggested that personnel engaged in the catering industry should earnestly carry out pre-employment medical examinations, frequently attend food hygiene training and develop good hygiene practices; long-term monitoring should be carried out in patients previously infected with Shigella for prevention and timely treatment of reinfection, especially those who live in a closed or semi closed environment.

\section{Abbreviations}

S. flexneri: Shigella flexneri; PFGE: Pulsed-field gel electrophoresis; ESBL: extended-spectrum $\beta$-lactamase; QRDRs: quinolone resistance determining regions

\section{Declarations}

\section{Acknowledgments}

We thank all the staff involved in this outbreak investigation.

\section{Funding}

This work was supported by the Zhejiang Medical and Health Science and Technology Project (grant no. 2015KYA194). The funding bodies had no influence in the design of this study or the collection, analysis, or interpretation of data as well as in writing the manuscript.

\section{Availability of data and materials}

All data generated or analyzed during this study are included in this published article

\section{Authors' contributions}

CLZ designed the study, performed the experiments, analyzed the clinical data and wrote the manuscript; TTL, CY, and YYG collected the clinical samples and investigated the case. All authors read and approved the final manuscript.

\section{Ethics approval and consent to participate}

Our study was approved by the Ethics Review Committee of the Mental Healthcare Center of Xiaoshan District. The guardians of all these patients with mental illness provided consent on their behalf.

\section{Consent for publication}

Not applicable.

\section{Competing interests}

The authors declare that they have no competing interests. 


\section{Author details}

Department of Clinical Laboratory, Zhejiang Xiaoshan Hospital, Hangzhou, People's Republic of China(Tiantian Lu, Chuanling Zhang, Yan Chen, Yuanyu Guo)

*Corresponding author:

Chuanling Zhang, Email: wuhuigt@163.com

Institution and address: Department of Clinical Laboratory, Zhejiang Xiaoshan Hospital, 728 Yucai North Rd., Hangzhou, Zhejiang Province, People's Republic of China; Zip Code: 311202

\section{References}

1. GBD 2015 Mortality and Causes of Death Collaborators. Global, regional, and national life expectancy, all-cause mortality, and cause-specifc mortality for 249 causes of death, 1980-2015: a systematic analysis for the Global Burden of Disease Study 2015. Lancet 2016; 388 (10053): 1459544.

2. Kotloff KL, Nataro JP, Blackwelder WC, Nasrin D, Farag TH, Panchalingam S, et al. Burden and aetiology of diarrhoeal disease in infants and young children in developing countries (the Global Enteric Multicenter Study, GEMS): a prospective, case-control study. Lancet 2013; 382(9888): 20922.

3. Chang Z, Lu S, Chen L, Jin Q, Yang J. Causative species and serotypes of shigellosis in mainland China: systematic review and meta-analysis. PLoS One 2012; 7(12): e52515.

4. Debnath F, Mukhopadhyay AK, Chowdhury G, Saha RN, Dutta S. An Outbreak of Foodborne Infection Caused by Shigella sonnei in West Bengal, India. Jpn J Infect Dis 2018; 71(2): 162-6.

5. Reller ME, Nelson JM, Mølbak K, Ackman DM, Schoonmaker-Bopp DJ, Root TP, et al. A large, multiplerestaurant outbreak of infection with Shigella flexneri serotype $2 a$ traced to tomatoes. Clin Infect Dis 2006; 42(2): 163-9.

6. Kim JS, Kim JJ, Kim SJ, Jeon SE, Seo KY, Choi JK, et al. Outbreak of Ciprofloxacin-Resistant Shigella sonnei Associated with Travel to Vietnam, Republic of Korea. Emerg Infect Dis 2015; 21(7): 1247-50.

7. Shen H, Chen J, Xu Y, Lai Z, Zhang J, Yang H, et al. An outbreak of shigellosis in a Children Welfare Institute caused by a multiple-antibiotic-resistant strain of Shigella flexneri $2 \mathrm{a} . \mathrm{J}$ Infect Public Health 2017; 10(6): 814-8.

8. Kozyreva VK, Jospin G, Greninger AL, Watt JP, Eisen JA, Chaturvedi V. Recent Outbreaks of Shigellosis in California Caused by Two Distinct Populations of Shigella sonnei with either Increased Virulence or Fluoroquinolone Resistance. mSphere 2016 ;1(6): e00344-16.

9. Pilon PA, Camara B, Bekal S. Outbreak of Shigella sonnei in Montréal's ultra-Orthodox Jewish community, 2015. Can Comm Dis Rep 2016; 42(4): 89-95. 
10. Bardhan P, Faruque AS, Naheed A, Sack DA. Decrease in shigellosis-related deaths without Shigella spp.-specific interventions, Asia. Emerg Infect Dis 2010; 16(11): 1718-23.

11. ATaneja N, Mewara A. Shigellosis: epidemiology in India. Indian J Med Res 2016; 143(5): 565-576.

12. Bhattacharya D, Bhattacharya $H$, Sayi DS, Bharadwaj AP, Singhania M, Sugunan AP, et al. Changing patterns and widening of antibiotic resistance in Shigella spp. over a decade (2000-2011), Andaman Islands, India. Epidemiol Infect 2015; 143(3):470-7.

13. Hassing RJ, Melles DC, Goessens WH, Rijnders BJ. Case of Shigella flexneri infection with treatment failure due to azithromycin resistance in an HIV positive patient. Infection 2014; 42(4): 789-90.

14. Zhang CL, Liu QZ, Wang J, Chu X, Shen LM, Guo YY. Epidemic and virulence characteristic of Shigella $s p p$. with extended-spectrum cephalosporin resistance in Xiaoshan District, Hangzhou, China. BMC Infectious Diseases 2014; 14: 260.

15. Zhao WH, Hu ZQ. Epidemiology and genetics of CTX-M extended-spectrum $\beta$-lactamases in Gramnegative bacteria.Crit Rev Microbiol 2013; 39(1): 79-101.

16. Novais A, Cantón R, Coque TM, Moya A, Baquero F, Galán JC. Mutational events in cefotaximase extended-spectrum beta-lactamases of the CTX-M-1 cluster involved in ceftazidime resistance. Antimicrob Agents Chemother 2008; 52(7): 2377-82.

17. Folster JP, Pecic G, Bowen A, Rickert R, Carattoli A, Whichard JM. Decreased susceptibility to ciprofloxacin among Shigella isolates in the United States, 2006 to 2009. Antimicrob Agents Chemother 2011; 55(4): 1758-60.

18. Sousa MÂ, Mendes EN, Collares GB, Péret-Filho LA, Penna FJ, Magalhães PP. Shigella in Brazilian children with acute diarrhoea: prevalence, antimicrobial resistance and virulence genes. Mem Inst Oswaldo Cruz 2013; 108(1): 30-35.

19. Yoshida S, Handa Y, Suzuki T, Ogawa M, Suzuki M, Tamai A, et al. Microtubule-severing activity of Shigella is pivotal for intercellular spreading. Science 2006; 314(5801): 985-9.

20. Schroeder G区Hilbi H. Molecular Pathogenesis of Shigella spp.: Controlling Host Cell Signaling $\rrbracket$ Invasion and Death by Type III Secretion. Clinical Microbiology Reviews 2008; 21(1): 134-156.

21. Clinical and Laboratory Standards Institute: Performance standards for antimicrobial susceptibility testing; Twenty-fourth Informational supplement, Document M100-S24. Wayne, PA: CLSI; 2014.

22. Dutta S, Kawamura Y, Ezaki T, Nair GB, lida K, Yoshida S. Alteration in the GyrA subunit of DNA gyrase and the ParC subunit of topoisomerase IV in Quinoloneresistant Shigella dysenteriae serotype 1 clinical isolates from Kolkata, India. Antimicrob Agents Chemother 2005; 49(4): 1660-1.

23. Ribot EM, Fair MA, Gautom R, Cameron DN, Hunter SB, Swaminathan B, et al. Standardization of pulsed-field gel electrophoresis protocols for the subtyping of Escherichia coli 0157: H7, Salmonella, and Shigella for PulseNet. Foodborne Pathog Dis 2006; 3(1): 59-67.

\section{Tables}

Table 1 
MICs for the outbreak Shigella flexneri 2a isolates.

\begin{tabular}{llllllllllll}
\hline Isolation no. & \multicolumn{10}{c}{ MICs $(\mu \mathrm{g} / \mathrm{mL})$} \\
\cline { 2 - 11 } & AMP & CIP & SXT & FEP & CTX & CAZ & IPM & TZP & AZM & TET \\
\hline jw001 & $>256$ & $>32$ & $>4$ & 128 & $>256$ & $>256$ & 0.5 & 0.5 & 2 & $>64$ \\
jw002 & $>256$ & $>32$ & $>4$ & 128 & $>256$ & $>256$ & 0.5 & 0.5 & 2 & $>64$ \\
jw003 & $>256$ & $>32$ & $>4$ & 128 & $>256$ & $>256$ & 0.5 & 0.5 & 2 & $>64$ \\
jw004 & $>256$ & $>32$ & $>4$ & 128 & $>256$ & $>256$ & 0.5 & 0.5 & 2 & $>64$ \\
jw005 & $>256$ & $>32$ & $>4$ & 128 & $>256$ & $>256$ & 0.5 & 0.5 & 2 & $>64$ \\
jw006 & $>256$ & $>32$ & $>4$ & 128 & $>256$ & $>256$ & 0.5 & 0.5 & 2 & $>64$ \\
jw007 & $>256$ & $>32$ & $>4$ & 128 & $>256$ & $>256$ & 0.5 & 0.5 & 2 & $>64$ \\
jw008 & $>256$ & $>32$ & $>4$ & 128 & $>256$ & $>256$ & 0.5 & 0.5 & 2 & $>64$ \\
jw009 & $>256$ & $>32$ & $>4$ & 128 & $>256$ & $>256$ & 0.5 & 0.5 & 2 & $>64$ \\
\hline
\end{tabular}

AMP, ampicillin; CIP, ciprofloxacin; SXT, trimethoprim/sulfamethoxazole; FEP, cefepime; CTX, cefotaxime; CAZ, ceftazidime; IPM, imipenem; TZP, piperacillin/tazobactam; AZM, azithromycin; TET, tetracycline

Table 2

Characterization of resistance genes and virulence genes of the outbreak Shigella flexneri 2a isolates

\begin{tabular}{|c|c|c|c|c|c|c|}
\hline \multirow{2}{*}{$\begin{array}{c}\text { Isolation } \\
\text { No. }\end{array}$} & \multicolumn{3}{|c|}{ ESBL-genes } & \multicolumn{2}{|c|}{ mutation in QRDR } & \multirow[t]{2}{*}{ Virulence genes } \\
\hline & CTX-M & OXA & TEM & gyrA & parC & \\
\hline jw001 & CTX-M-57 & OXA-30 & TEM-1 & Ser83Leu & Ser80Ile & vira-ial-ipaH- set1B-sen \\
\hline jw002 & CTX-M-57 & OXA-30 & TEM-1 & Ser83Leu & Ser80Ile & vira-ial-ipaH- set1B-sen \\
\hline jw003 & CTX-M-57 & OXA-30 & TEM-1 & Ser83Leu & Ser80Ile & vira-ial-ipaH-set1B-sen \\
\hline jw004 & CTX-M-57 & OXA-30 & TEM-1 & Ser83Leu & Ser80Ile & vira-ial-ipaH-set1B-sen \\
\hline jw005 & CTX-M-57 & OXA-30 & TEM-1 & Ser83Leu & Ser80Ile & vira-ial-ipaH-set1B-sen \\
\hline jw006 & CTX-M-57 & OXA-30 & TEM-1 & Ser83Leu & Ser80Ile & vira-ial-ipaH-set1B-sen \\
\hline jw007 & CTX-M-57 & OXA-30 & TEM-1 & Ser83Leu & Ser80Ile & vira-ial-ipaH-set1B-sen \\
\hline jw008 & CTX-M-57 & OXA-30 & TEM-1 & Ser83Leu & Ser80Ile & vira-ial-ipaH-set1B-sen \\
\hline jw009 & CTX-M-57 & OXA-30 & TEM-1 & Ser83Leu & Ser80Ile & vira-ial-ipaH-set1B-sen \\
\hline
\end{tabular}

\section{Figures}




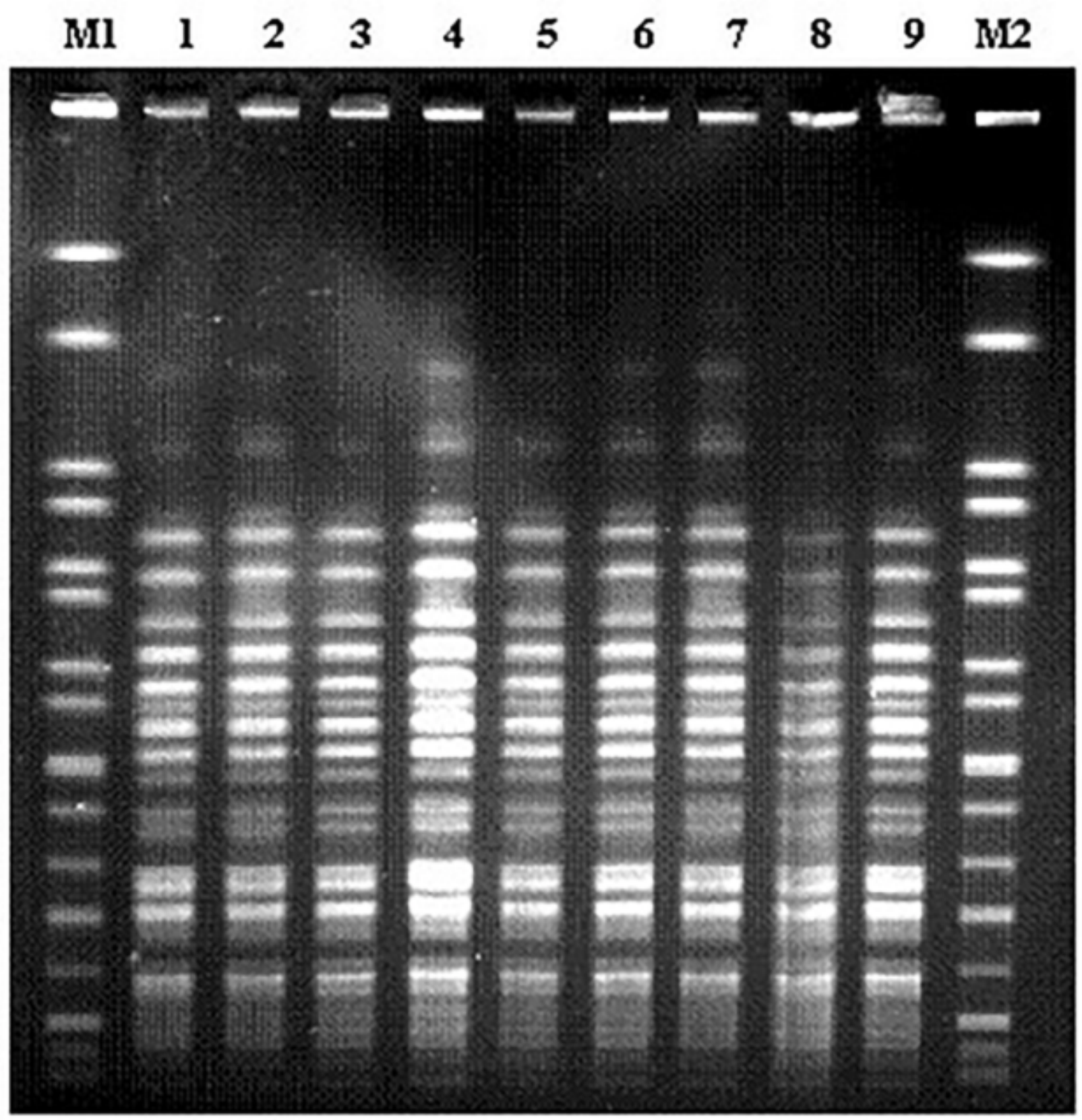

Figure 1

The pulsed-field gel electrophoresis patterns of 9 isolates. Lane M1, M2, molecular mass markers. Line 18, patient. Line 9, canteen staff 